\title{
Estigma e tuberculose: olhar dos agentes comunitários de saúde
}

\author{
Lílian Moura de Lima, ${ }^{1}$ Roxana Isabel Cardozo-Gonzales, ${ }^{2}$ Eda Schuwartz, ${ }^{3}$ Ludmila Meireles Costa, ${ }^{4}$ \\ Dagoberta Alves Vieira Beduhn, ${ }^{5}$ Jéssica Tomberg ${ }^{6}$
}

\author{
Lima LM, Cardozo-Gonzales RI, Schuwartz E, Costa \\ LM, Beduhn DAV, Tomberg J. Estigma e \\ tuberculose: olhar dos agentes comunitários de \\ saúde. Cuid salud, ene-jun 2014; 1(1).
}

\section{RESUMO}

Este estudo objetivou discutir o estigma e suas implicações para o cuidado e o controle da tuberculose sob a perspectiva dos agentes comunitários de saúde de um município do sul do Rio Grande do Sul. A coleta de dados foi realizada por meio da aplicação de entrevista semiestruturada, com a participação de 12 sujeitos. Os dados foram analisados conforme a técnica de análise de conteúdo modalidade temática. Os resultados evidenciam a presença do estigma no processo de cuidado e suas implicações para a assistência e o controle da tuberculose. Considerase que o estigma sobre a pessoa com tuberculose é um desafio para a realização do cuidado integral e de qualidade para o controle da doença. Portanto, é imprescindível a mudança no posicionamento e na abordagem dos profissionais de saúde no processo de cuidado às pessoas com Tuberculose.

Descriptores: tuberculose, preconceito, programa saúde da família.

\begin{abstract}
Lima LM, Cardozo-Gonzales RI, Schuwartz E, Costa LM, Beduhn DAV, Tomberg J. Stigma and tuberculosis: according to the view professionals of primary health care. Cuid salud, ene-jun 2014; 1(1).
\end{abstract}

\begin{abstract}
This research had the objective to discuss stigma and its implications for care and control of tuberculosis from the perspective of community health agents of a city in southern of Rio Grande do Sul. Data collection was performed through the application of semi-structured interview to 12 subjects. The data were analyzed using thematic technique of content analysis. The results show the presence of stigma in the care process and its implications for the care and control of tuberculosis. It is understood that stigma on the person with tuberculosis is a challenge for a comprehensive and high quality care for the control of the disease. It is essential to change the positioning and the approach's form from health professionals in the process of care on people with Tuberculosis.
\end{abstract}

Keywords: tuberculosis, prejudice, family health program.

\section{RESUMEN}

Este estudio tuvo como objetivo discutir el estigma y sus implicancias para el cuidado y el control de la tuberculosis en la perspectiva de los promotores de salud en un municipio del sur de Rio Grande do Sul. La recolección de datos fue realizada mediante la aplicación de entrevista semiestructurada, con participación de 12 promotores de salud. Los datos se analizaron utilizando la técnica de análisis de contenido, modalidad temática. Los resultados revelan presencia del estigma en el proceso de cuidado y sus implicancias para la asistencia y control de la tuberculosis. Se considera que la presencia de estigma en la persona con tuberculosis es un desafío para la realización del cuidado integral y de calidad para el control de la enfermedad. Por lo tanto, es imprescindible cambios en el posicionamiento y en el abordaje de los profesionales de salud a las personas con tuberculosis en el proceso de cuidado.

Palabras clave: tuberculosis, preconcepto, programa salud de la familia.

\footnotetext{
${ }^{1}$ Doutoranda do Programa DE Pós Graduação da Faculdade de Enfermagem/Universidade Federal de Pelotas (UFPel), Rio Grande do Sul-Brasil, ${ }^{2}$ Doutoranda em Saúde Pública. Docente da Faculdade de Enfermagem/UFPel, Rio Grande Do Sul-Brasil, ${ }^{3}$ Doutoranda em Enfermagem. Docente da Faculdade de Enfermagem/UFPel, Rio Grande do Sul-Brasil, ${ }^{4}$ Enfermeira. Secretaria Municipal de Rio Grande - Rio Grande do Sul-Brasil, ${ }^{5}$ Mestranda do Programa de Pós Graduação da Faculdade de Enfermagem/UFPel, Rio Grande do Sul-Brasil, ${ }^{6}$ Doutoranda do Programa de Pós Graduação da Faculdade de Enfermagem/UFPel, Rio Grande do Sul-Brasil..
} 


\section{INTRODUÇÃO}

A tuberculose se mantém como um problema de saúde pública mundial. Vários fatores de origem social, econômico e cultural influenciam o processo de adoecimento e determinam a grande magnitude, transcendência e vulnerabilidade da doença, o que a torna prioridade, merecendo atenção especial, por parte do governo, dos profissionais da saúde e da população em geral. ${ }^{1}$

A doença possui raízes sociais e tem relação diretamente proporcional com a pobreza, considerando-se que as precárias condições de alimentação e moradia contribuem para o seu desenvolvimento. ${ }^{2}$ Este panorama torna-se ainda mais adverso por atribuir-se à tuberculose o juízo de punição social, o que a mantém como estigmatizante, condicionando à pessoa a uma situação de isolamento social, mesmo após o período de transmissibilidade da doença. ${ }^{3}$

A tuberculose, historicamente é envolvida por estigma, carregada de culpabilidade, medo da contaminação e da morte. ${ }^{4} \mathrm{O}$ termo estigma, de origem grega, é amplamente utilizado no seu sentido original literal (sinais corporais com os quais se procurara evidenciar alguma coisa de extraordinário ou mau sobre o estado moral de quem o apresenta). Considera-se como a presença de uma ou várias características que podem colocar o indivíduo em uma condição de normalidade ou não, dependendo da compreensão da sociedade. ${ }^{5}$ Contudo, o termo é mais utilizado em sua interpretação negativa. Há determinadas características que coloca o indivíduo em uma condição depreciativa pela maioria das pessoas, como o ser doente de tuberculose.

Não é incomum atribuir-se um juízo negativo à pessoa doente de tuberculose, o que vem contribuir para a exclusão e o isolamento deste indivíduo, devido a exposição pública ${ }^{6}$ no processo de diagnóstico e tratamento. Nessa perspectiva, muitas vezes, a pessoa só procura o serviço de saúde quando desenvolve sintomas mais graves, retardando o diagnóstico e o início do tratamento, elevando o risco de transmissão da tuberculose. Entende-se que conflitos nos relacionamentos sociais da pessoa com tuberculose estimulam o estigma, o preconceito e o afastamento da vida social. ${ }^{3}$

Visando a superação do distanciamento entre usuários e serviços de saúde é esperado que a Estratégia Saúde da Família, por meio do agente comunitário de saúde (ACS), por ser membro do território, seja capaz de construir um elo entre os serviços de saúde e a comunidade. Este profissional deve ser capaz de identificar os sintomáticos respiratórios na comunidade, encaminhando-os à unidade de saúde do território. Em articulação com os demais profissionais, o ACS também é responsável por orientações às famílias e a comunidade, além de acompanhar o tratamento medicamentoso dos casos positivos. ${ }^{1}$ Diante disto, é importante que o profissional esteja preparado para desenvolver ações educativas que desmistifiquem a tuberculose na população, uma vez que, o estigma sentido pelo doente constitui-se uma importante barreira na busca por cuidados de saúde. $^{7}$

Contudo o presente artigo se insere nesse contexto de discussão, como uma possível contribuição aos profissionais da saúde no cotidiano de trabalho para a assistência as pessoas com tuberculose. Espera-se estimular a reflexão sobre a necessidade do compromisso do profissional com a reintegração destes sujeitos na sociedade, reduzindo as barreiras do preconceito, do estigma e do medo.

Nesse sentido, este estudo objetivou discutir o estigma e suas implicações para o cuidado e o controle da tuberculose sob a perspectiva dos agentes comunitários de saúde de um município do sul do Rio Grande do Sul.

\section{METODOLOGIA}

Trata-se de um estudo qualitativo descritivo, que discute a temática em estudo sob o conceito de estigma referido por Goffman, ${ }^{5}$ realizado em município de médio porte localizado no Sul do Brasil, considerado prioritário para o controle da tuberculose. Este manuscrito é produto de um trabalho monográfico que abordou o controle da tuberculose na estratégia de saúde da família. ${ }^{8}$

No município em estudo ocorrem em média 200 casos novos de tuberculose. A atenção à doença é centralizada no Programa de Controle da Tuberculose (PCT), dessa forma, as unidades de atenção básica à saúde são responsáveis pelas ações de busca de sintomáticos respiratórios e detecção de casos para encaminhamento ao PCT. A unidade em estudo está situada em um bairro de população com características de vulnerabilidade social, possuindo uma equipe composta por 23 profissionais, sendo 13 agentes comunitários de saúde. 
Participaram deste estudo os 12 agentes comunitários de saúde, (em exercício no momento das entrevistas) que integravam as quatro equipes atuantes na unidade de Saúde da Família, selecionada em virtude da elevada ocorrência dos casos de tuberculose em seu território. Nesse estudo não houve nenhuma recusa à participação nas entrevistas. O critério de inclusão foi ter cinco anos de experiência profissional na unidade em estudo; sendo excluídos os ACS que estavam afastados no período da entrevista por férias ou benefício saúde.

A coleta de dados ocorreu no mês de julho de 2010, na unidade de saúde em estudo, sendo aplicada uma entrevista semiestruturada, previamente agendada, em local reservado e com aproximadamente 30 minutos de duração. Utilizou-se gravador digital para posterior transcrição das falas na íntegra. Como estratégia para preservar a identidade dos sujeitos foi atribuída nomes fictícios.

Para organização dos resultados foi utilizada análise de conteúdo de Minayo. ${ }^{9}$ Salienta-se que os ACS foram questionados a respeito de: "Quais ações de controle são executadas diante as pessoas com tuberculose?". A categoria de análise utilizada para a construção deste estudo emergiu espontaneamente na fala dos entrevistados, e foi explorada devido a sua relevância diante o adoecimento por tuberculose. Sendo, "O estigma no processo de atenção à pessoa com tuberculose". Finalizou-se a análise dos dados com a interpretação e a discussão do conteúdo da categoria com a literatura atual sobre o assunto.

A pesquisa respeitou os preceitos recomendados pela Resolução do Conselho Nacional de Saúde no 466/2012. ${ }^{10}$ Foi submetida à apreciação no Comitê de Ética e Pesquisa da Faculdade de Enfermagem - Universidade Federal de Pelotas (UFPel), obtendo parecer favorável sob $n^{\circ} 116 / 2010$. Os sujeitos foram esclarecidos sobre seus direitos de desistir a qualquer momento da entrevista assim como a garantia do anonimato. Todos eles assinaram o Termo de Consentimento Livre e Esclarecido.

\section{RESULTADOS}

As falas dos profissionais de saúde revelaram a presença do estigma no processo de atenção às pessoas com tuberculose, identificando as suas implicações para o cuidado e o controle da doença, bem como, as intervenções realizadas pela equipe de saúde para reduzi-lo. No discurso dos entrevistados identifica-se que a pessoa com tuberculose possui atributos depreciativos da doença que nos remetem à presença do estigma da tuberculose:

[...] tem muitas pessoas que tem vergonha, porque estão com suspeita de tuberculose. [...] existe preconceito sim, com as pessoas que tem tuberculose (Alice, ACS).

Os agentes comunitários reconhecem que estar doente de tuberculose e relacioná-lo com significado depreciativo, é uma carga pesada que gera medo e leva ao isolamento social, piora das condições socioeconômicas, tornando difícil a reinserção da pessoa na vida em sociedade:

[...] eles têm medo que as pessoas vão se afastar deles, vão se escondendo até a unidade, com medo que alguém conhecido veja, e diga que ele está com tuberculose (Dora, ACS).

[...] eles têm, aquele preconceito, não querem falar, porque as pessoas vão se afastar o preconceito ainda existe hoje, apesar de a mídia falar muito, fazer campanhas, aquela coisa toda, mas ainda tem [...] (Georgete, ACS).

[...] ela não quer que ninguém saiba, nem a família dela sabe [...] quando vai à unidade de saúde, pensa, eu to aqui, todo mundo já tá me olhando, porque eles se sentem diferentes dos outros pacientes que estão ali (Suzana, ACS).

No relato dos profissionais de saúde, emerge os sentimentos e as estratégias utilizadas pelos sujeitos estigmatizados, neste caso a pessoa com tuberculose, tais como o isolamento social e familiar, ao medo de ser descoberto com a doença e a omissão das informações sobre os sintomas da doença, como uma forma de autoproteção. Este último mecanismo é utilizado nas relações com os profissionais, com a sociedade e com a própria família.

Percebe-se que a discriminação ocorre dentro da própria família e nos profissionais de saúde. Nesse sentido, é desencadeado um conjunto de atitudes no interior da família como citado na fala do entrevistado:

[...] eles já sabem qual é o problema deles então eles já sentem em casa o preconceito da família, aquele negócio de separar as coisas, já com aquilo ele se sente até humilhado [...] (Suzana, ACS). 
Tais atitudes refletem a presença do medo do contágio da doença, fato que leva a família a adotar medidas de prevenção inapropriadas. Esse comportamento pode potencializar o sofrimento causado pelo estigma da pessoa com tuberculose e acarretar maior isolamento e sentimento de exclusão.

Apesar das informações disponíveis sobre a doença e da vivência do profissional no cotidiano da prática, o discurso a seguir transparece uma dificuldade do mesmo na relação profissionalusuário:

[...] é a questão do preconceito dos próprios profissionais. Porque, nós assim trabalhando na área já há bastante tempo, a gente já presenciou o preconceito dos profissionais, que mesmo com toda aquela bagagem de informação, de como que se pega, em que ponto o bacilo vai [...] mas na hora que ele se depara com o problema, ele não consegue, ele trava, não consegue abordar, conversar com o paciente normalmente, sem aquele preconceito (Cláudia, ACS).

Embora na fala do entrevistado cita-se o acesso à informação sobre a tuberculose, isto não é o suficiente para qualificar a interação do profissional de saúde com a pessoa doente. Tal realidade pode estar atrelada à existência de um juízo negativo e/ou ao medo do contágio e inclusive ao despreparo profissional para lidar com a doença e para acolher os adoecidos nos serviços de atenção básica, conforme trecho:

[...] eu acho que aqui na unidade o pessoal não tá preparado para receber as pessoas com tuberculose, porque se chegar alguém aqui com sintomas, tossindo, os próprios funcionários já ficam se cuidando (Jussara, ACS).

A manifestação da discriminação, em função do estigma do adoecido por tuberculose pelos profissionais, apresenta singularidades em função da categoria profissional e do conjunto de atividades inerentes ao cargo e à forma de organização do serviço de saúde. Percebe-se que o ACS vivência o cuidado à pessoa estigmatizada de forma tênue em função da sua inserção na comunidade, o que pode ser percebido na fala:

[...] nós (agente comunitário de saúde) por estarmos lá dentro da residência deles, não vamos ter tanto preconceito. Então eles pedem muito isso, a nossa busca é essa, chegar nessa casa, conversar com eles, trazer eles até a unidade (Alice, ACS).
Em decorrência do estigma da tuberculose, que provoca preconceito e discriminação por parte dos profissionais de saúde, fragiliza-se a humanização do atendimento, pautada na relação profissional-usuário, no acolhimento e na escuta qualificada, esses necessários para as ações de cuidado. O contato ocorre de forma rápida sem espaço para formação de vínculo, como revela a fala a seguir:

[...] eles nos passam que se sentem rejeitados, se sentem tratado diferente. A maneira que o profissional tá falando com ele, sentem uma diferença no tratamento, então na verdade eles querem expor ali os seus problemas, conversar, querem que alguém apenas escute eles ali, eles vêem que não existe isso, esse vínculo com o profissional da saúde ali, não tem isso, justamente pelo preconceito, a pessoa se fecha, ela trata ali como se fosse só mais uma, em 5 minutinhos e deu, tu precisa disso e daquilo, tchau, vai com Deus, vira as costas, então não existe esse vínculo, assim, da pessoa poder se abrir com o profissional, e isso é uma dificuldade que eles encontram (Claudia, ACS).

Na visão dos ACS, a tuberculose, doença infecto-contagiosa que pode ser transmitida facilmente pelo contato com o outro, é encarada como um quadro semelhante das demais doenças acompanhadas pela equipe. Não há prioridades ou acolhimento específico a despeito dos riscos, como fica claro no depoimento a seguir:

[...] pacientes que já sabem que são portadores, chegam na sala de espera, e eles não tem uma prioridade, de chegar e ir direto com o médico, eles já ficam naquela preocupação, [...] todo mundo está me olhando, porque eles se sentem diferentes dos outros pacientes que estão ali, até porque eles já sabem qual é o problema deles (Suzana, ACS).

O estigma da tuberculose tem implicações negativas no processo de diagnóstico, uma vez que a pessoa adoecida, ao percebê-lo, muitas vezes, opta pelo silêncio, sentindo-se coagido a mantê-lo, ao invés de tornar-se voz ativa e pedir por ajuda. Nesse sentido, forma-se uma cascata de equívocos, que vão desde a desinformação do usuário, passando pela organização das práticas dos serviços, até o seu afastamento do convívio social:

[...] a dificuldade na detecção dos casos é em relação ao paciente, aquele bloqueio que eles têm, não querem falar [...] (Georgete, ACS). 
[...] ela não quer que ninguém saiba [...] nem a família dela sabe então eu e a enfermeira conversamos com ela, ela esteve aqui e ninguém ficou sabendo, nem na unidade, nem na casa dela, ela nos pediu sigilo (Suzana, ACS).

Diante da presença do estigma da tuberculose, os entrevistados relatam que a estratégia utilizada para enfrentá-lo seria a sensibilização pautada no conhecimento da doença, de seus sintomas, do tratamento e das formas de contágio:

[...] existe muito preconceito, então teria que ter uma ação de saúde, de todo o município em que sensibilizasse os profissionais e a comunidade, para que a população interaja com os profissionais e desmistifique a tuberculose. Porque quanto mais conhecimento a população tiver sobre o que é tuberculose, quais os sintomas e que com tratamento não há contágio, tratamento correto, que tem cura, além de conscientizar vai diminuir o número de casos (Eugênia, ACS).

Os depoimentos apontam as consequências do estigma atribuído às pessoas com tuberculose, $\mathrm{o}$ que reflete atitudes de discriminação, afastamento, desumanização, despersonalização do atendimento no processo de cuidado e de diagnóstico da tuberculose, salientando as repercussões no bem estar físico e emocional da pessoa doente e no desenvolvimento de ações de saúde voltadas para o diagnóstico precoce da doença.

\section{DISCUSSÃO}

A vivência do adoecer sofre interferência das diversas fantasias que o indivíduo faz de si mesmo e da maneira como se relaciona com a realidade, fazendo com que esta experiência adquira uma conotação ora solitária, ora coletiva por todas as transformações que desencadeia em seu meio social. $^{11}$

Há um intrincado conjunto de elementos subjetivos que atuam no adoecer do indivíduo, dentre os quais estão os sentimentos que o enfermo associa a cada sintoma que apresenta, a forma como tomou conhecimento da doença que o acomete, as experiências passadas, a maneira como as pessoas do seu meio interagem e se relacionam com o adoecimento, e como os reflexos deste comportamento permanecem na história em questão. $^{11}$ Toda esta complexa problemática interfere nas relações da pessoa com tuberculose com a sua família, com a sociedade e com os serviços de saúde.
Ao longo da história, a representação da tuberculose é marcada pelo preconceito e o estigma. ${ }^{12}$ Ainda hoje, a tuberculose é temida por ser a expressão de algo que é socialmente digno de censura, bem como, por representar o estágio último de miséria humana. Nesse sentido, persiste no imaginário social e como forma de relação da sociedade com a pessoa com tuberculose, o estigma sobre a tuberculose e a pessoa adoecida. ${ }^{13}$

O estigma se institui na relação entre a cultura e o poder. Quando valorizada negativamente age como promotor da desigualdade social. ${ }^{12}$ A estigmatização inicia-se na identificação de uma característica particular como sendo indesejável, ou fora dos padrões de determinada sociedade, passando o seu portador a ser identificado por um estereótipo indesejável, que resulta em tratamento discriminatório. ${ }^{6}$

$\mathrm{O}$ indivíduo ou grupo que possui uma ou várias características indesejáveis, muitas vezes, internaliza um sentimento pejorativo e adota atitudes decorrentes da auto-percepção sobre esta particularidade que podem incluir vergonha, nojo e culpa. Estes sentimentos produzem um conjunto de comportamentos que vão desde a negação do traço estigmatizado e a exclusão social até o aumento do comportamento de risco. ${ }^{14}$

Tais situações são percebidas pelo ACS e foi revelado em suas falas que é necessário promover o acesso a informações sobre a doença para a população, que esse conhecimento extrapole os muros das unidades de saúde, isto é que seja disponibilizado para as pessoas, como já citado pela literatura científica, a qual afirma que a tuberculose vem sendo considerada, por muitos, como um problema do passado, facilmente resolvível através do conhecimento produzido sobre a doença ao longo dos tempos. Entretanto, desconsidera-se que a existência desse saber por si só não garante a cura e o controle da doença, é necessário que este conhecimento seja disponibilizado a toda a população ${ }^{7,14,15}$ uma vez que, esse saber, muitas vezes, por diversos fatores, restringem-se ou concentram-se em espaços específicos (gestores, academia e profissionais de saúde) que não o da sociedade em geral.

Além disso, a deficiência de informação favorece o fortalecimento de sentimentos, como o medo do contágio pela doença, o qual pode ser percebido na fala dos ACS. O significado depreciativo sentido pelo doente por múltiplos 
fatores desencadeia mecanismos de proteção geralmente inadequados por se constituírem em comportamentos de risco, tais como, o isolamento social ou mesmo o silêncio para o ocultamento da doença, na tentativa de usar um disfarce para ser aceito pelos demais. ${ }^{5}$ Os enfermos, muitas vezes, se isolam de seu grupo familiar e social para evitar infectá-los e fugir de situações desconfortáveis, tais como, tornar-se objeto de rumores e de hesitação dos amigos e familiares, sentindo-se coagido a comer e dormir separadamente do restante da família. ${ }^{6,16}$

$\mathrm{Na}$ tentativa de evitar o estigma da tuberculose e, o isolamento dele decorrente, o enfermo nega a doença, atribuindo aos sintomas da doença a outras patologias não contagiosas. ${ }^{17}$ Esta afirmação pode ser percebida no discurso dos entrevistados, quando se referem aos relatos concedidos pelas pessoas com tuberculose, durante visitação domiciliar, sobre a rejeição sentida em sua rotina familiar e social após o adoecimento.

Estas confidências confirmam que a participação dos profissionais de saúde é importante no processo de adoecer da pessoa com tuberculose. Entretanto, percebe-se que o medo do contágio e/ou o estigma da doença influencia no atendimento dispensado às pessoas com a tuberculose, sendo muitas vezes negligenciada e omitida pelos profissionais. ${ }^{11}$ Diante disso, o desconhecimento dos sinais e sintomas característicos da tuberculose, assim como, as formas de transmissão da doença por partes dos ACS, coloca em risco a efetividade de suas ações, sendo necessário manter a qualificação continuada destes profissionais. ${ }^{18}$

Nesse sentido, os ACS referem que, também na sua comunidade, a assistência é prestada de forma rápida e superficial para evitar o contato prolongado com o doente, comprometendo a qualidade do cuidado e caracterizando o despreparo técnico dos profissionais, sendo agravada pela adoção de precauções exageradas e desatualizadas, que fomentam o preconceito, o medo e o estigma, afastando a pessoa com tuberculose dos serviços de saúde. ${ }^{16}$

Esperam o oposto disso, uma prática de saúde pautada no vínculo e no acolhimento humanizado, essenciais no processo de atenção à pessoa com tuberculose. As dúvidas do doente a respeito da doença e seu tratamento, o medo, a vergonha e a culpa são sentimentos muitos presentes no processo de descoberta da doença, que precisam ser revelados e compartilhados, para um melhor enfrentamento da enfermidade. ${ }^{19}$

Outra questão ressaltada pelos ACS foi a importância do vínculo como facilitador da construção de relações favoráveis ao espaço de escuta de problemas e cuidado. Essa condição de interação cria um espaço seguro para que a pessoa revele sua vivência do adoecimento, solicitando, se for o caso, ajuda e cumplicidade no enfrentamento da tuberculose. Uma atenção com forte vínculo tem potencial para desencadear uma ação de saúde efetiva e capaz de estabelecer o diagnóstico precoce e o tratamento oportuno da doença, que além de contribuir no controle da tuberculose, promove uma justa reinserção social do enfermo. ${ }^{19}$

Outros estudos destacam que a realidade evidenciada na fala dos usuários de serviços de saúde, tem relação com a forma de atendimento recebido, principalmente no que diz respeito ao acolhimento e ao vínculo, as quais se concretizam na aplicação das tecnologias leves, caracterizadas pelo diálogo, que se inicia pelo simples chamamento do indivíduo pelo seu nome próprio e, no ato de cumprimentá-lo quando chega ao serviço. $^{20}$

Os ACS sinalizaram os aspectos relacionados aos conflitos intrafamiliares revelados pelas pessoas adoecidas de tuberculose. Apesar da reconhecida perturbação ocasionada pelo adoecimento de tuberculose no contexto familiar, ainda hoje, evidencia-se a dificuldade vivenciada pelo profissional de saúde ao abordar o indivíduo inserido em seu contexto sócio-cultural. Muitas vezes, enquanto um fala o outro filtra apenas as informações que lhe interessam do discurso, o que acaba sendo interpretado e utilizado de acordo com as necessidades próprias. $\mathrm{O}$ diálogo tende a virar um interrogatório, cujas perguntas nem sempre são esclarecidas e as respostas nem sempre levadas em conta no atropelo do atendimento nas unidades de saúde. ${ }^{10}$ Esta colocação pode ser percebida no discurso dos entrevistados, quando relatam a forma de atendimento oferecida pelos profissionais no serviço de saúde em estudo.

A ausência ou falta de conhecimento da doença, falhas na educação em saúde ou ações inexistentes nesse âmbito podem influenciar na preservação e fortalecimento do estigma e discriminação às pessoas com tuberculose. Em estudo realizado no Nepal, ${ }^{21}$ os respondentes relataram que o conhecimento da doença é deficiente e reportaram forte presença do estigma 
na comunidade. Relatam ainda que a existência do estigma associada à má compreensão da doença e aos problemas com a prestação de cuidados pelos serviços de saúde são as principais razões, para que as pessoas com a doença atrasem o diagnóstico e o tratamento da doença.

Diante do exposto percebe-se que o cuidado à pessoa com tuberculose requer necessariamente uma atenção integral e continua indo desde a perspectiva curativa até a compreensão social e cultural do adoecimento. A análise das iniquidades sociais e do direito à saúde é um importante assunto nesse complexo contexto de atuação dos profissionais de saúde, os quais podem e devem ter maior protagonismo na prevenção e no controle da doença. Desde que, alicercem a sua prática em uma ampla compreensão política, social e econômica da saúde, já que, a tomada de decisões especialmente do profissional ACS, que está, muitas vezes, mais próximo ao grupo familiar, pode favorecer o controle da tuberculose. ${ }^{22}$

As limitações deste estudo estiveram relacionadas à natureza dos dados se referirem a percepções dos ACS a respeito da situação vivenciada pelas pessoas com tuberculose, e não a observação efetiva da rotina de trabalho dos ACS. Dessa forma, as conclusões obtidas por este estudo referem-se ao que foi proferido pelos entrevistados

\section{Correspondência:}

Dagoberta Alves Vieira Beduhn

Rua Visconde de Sinimbú, N 526 - Centro

Pelotas, 96020-790

Correio electrônico: dagobertavieira@gmail.com

\section{REFERÊNCIAS BIBLIOGRÁFICAS}

1. Ministério da Saúde (BR), Secretaria de Vigilância em Saúde, Programa Nacional de Controle da Tuberculose. Manual de Recomendações para o Controle da Tuberculose no Brasil [internet]. Brasília (DF): Ministério da Saúde; 2010. [citado 2012 out 15]. Disponível em: http://portal.saude.gov.br/portal/arquivos/pdf/ manual_de_recomendacoes_controle_TB_nov o.pdf

2. Santos MLSG, Vendramini SHF, Gazetta CE, Oliveira SAC, Villa TCS. Pobreza: caracterização socioeconômica da tuberculose. Rev Lat-ame Enfermagem [internet]. Set-out 2007 [citado Set 24 de 2012]; 15(número especial): [06 laudas]. Disponível em: a cerca de suas vivencias, não podendo descrever as atividades efetivamente desenvolvidas no cotidiano das unidades de saúde pelos agentes comunitários. Para estudos futuros recomenda-se abordar a temática estigma sob a perspectiva da equipe de saúde da atenção básica, assim como, da pessoa com tuberculose e sua família, sendo possível ampliar a perspectiva de visualização da problemática.

Considera-se que a superação do estigma da tuberculose permanece como um desafio para a realização do cuidado integral com qualidade para o controle da doença. Dificuldades no acesso e na oferta de ações de saúde, muitas vezes são influenciadas pela presença do estigma, que por sua vez dificulta a prática dos princípios do Sistema Único de Saúde no que diz respeito à universalidade e a integralidade da atenção.

Entende-se que os ACS têm papel de protagonistas no controle da tuberculose, em virtude da sua proximidade com a vida na comunidade, inserindo-se no interior das residências e vivenciando a realidade dos usuários. Dessa forma, a qualificação destes atores para a atenção à doença torna-se indispensável para o alcance do efetivo controle da tuberculose e a integralidade da atenção.

$\underline{\text { http://www.scielo.br/pdf/rlae/v15nspe/pt_07.p }}$ df

3. Souza SS, Silva DMGV, Meirelles BHS. Representações sociais sobre a tuberculose. Acta Paul Enferm. 2010; 23(1):23-8.

4. Sontag S. A doença como metáfora. Rio de Janeiro: Edições Graal; 2002. 108 p.

5. Goffman E. Estigma: notas sobre a manipulação da identidade deteriorada. $4^{\mathrm{a}} \mathrm{ed}$. Rio de Janeiro: Guanabara Koogan; 1988. $124 \mathrm{p}$.

6. Baral SC, Karki DK, Newell JN. Causes of stigma and discrimination associated with 
tuberculosis in Nepal: a qualitative study. BMC Public Health [internet]. 2007 [citado Set 20 de 2012]; 7(211): [10 laudas]. Disponível em: http://www.biomedcentral.com/content/pdf/14 71-2458-7-211.pdf

7. Trigueiro JVS, Silva ACO, Gois GAS, Almeida SA, Nogueira JA, Sá LD. Percepção de enfermeiros sobre educação em saúde no controle da tuberculose. Cienc Cuid Saúde [internet]. Out-dez 2009 [citado out 02 de 2012]; 8(4):660-6. Disponível em: http://periodicos.uem.br/ojs/index.php/CiencC uidSaude/article/view/9697/5401

8. Costa LM. As ações de diagnóstico da tuberculose na atenção básica na visão dos profissionais de saúde [Monografia]. Pelotas: Universidade Federal de Pelotas, Curso de Enfermagem; 2010.

9. Minayo MCS. O desafio do conhecimento: pesquisa qualitativa em saúde. $11^{\mathrm{a}}$ ed. São Paulo: Hucitec; 2010. 408 p.

10. Ministério da Saúde (BR), Conselho Nacional de Saúde, Comissão Nacional de Ética em Pesquisa. Resolução $N^{\circ} 466$ de 2012: diretrizes e normas regulamentadoras de pesquisa envolvendo seres humanos. Brasília (DF): MS; 2012.

11. Pereira WSB, Lima CB. Tuberculose: sofrimento $\mathrm{e}$ ilusões no tratamento interrompido. Rev Bras Enferm. 1999; 52(2):303-18.

12. Clementino FS, Martiniano MS, Souza JC, Marcolino EC, Miranda FAN. Tuberculose: desvendando conflitos pessoais e sociais. Rev enferm UERJ [internet]. Out-dez 2011[citado set 30 de 2012];19(4):638-43. Disponível em: http://www.facenf.uerj.br/v19n4/v19n4a23.pd f

13. Pôrto A. Representações sociais da tuberculose: estigma e preconceito. Rev Saude Publica. 2007; 41(Supl. 1):43-49.

14. Courtwright A, Turner AN. Tuberculosis and stigmatization: pathways and interventions. Public Health Rep. 2010; 125(4): 34-42

15. Sá LD, Souza KMJ, Nunes MG, Palha PF, Nogueira JA, Villa TCS. Tratamento da tuberculose em unidades de saúde da família: histórias de abandono. Texto \& Contexto Enferm. 2007;16(4):712-8.

16. Gavíria MB, Henao HM, Martinez T, Bernal E. Papel del personal de salud en el diagnóstico tardío de la tuberculosis pulmonar en adultos de Medellín, Colombia. Rev Panam Salud Publica. 2010;27 (2):83-92.

17. Ngamvithayapong J, Winkvist A, Diwan V. High AIDS awareness may cause tuberculosis patient delay: results from an HIV epidemic area, Thailand. AIDS [internet]. 2000 [citado fev 10 de 2013]; 14(10):1413-19. Disponível em:

http://hawaii.edu/hivandaids/High_AIDS_Aw areness_May_Cause_Tuberculosis_Patient_D elay Results from an HIV Epidemic Area , Thailand.pdf

18. Maciel ELN, Vieira RCA, Milani EC, Brasil M, Fregona G, Dietze R. O agente comunitário de saúde no controle da tuberculose: conhecimentos e percepções. Cad. Saúde Pública. 2008; 24(6):1377-1386.

19. Brunello MEF, Cerqueira DF, Pinto IC, Arcênio RA, Gonzales RIC, Villa TCS, et al. Vínculo doente-profissional de saúde na atenção a pacientes com tuberculose. Acta Paul Enferm. 2009; 22(2):176-82.

20. Scholze AS, Duarte Junior CF, Flores e Silva Y. Trabalho em saúde e a implentação do acolhimento na atenção primária à saúde: afeto, empatia ou alteridade? Interface (Botucatu). 2009; 13(31):303-14.

21. Lewis PC, Newell J. Improving tuberculosis care in low income countries - a qualitative study of patients' understanding of "patient support" in Nepal. BMC Public Health. [internet]. 2009 [citado dez 08 de 2012]; 9 (190): [08 laudas]. Disponível em: http://www.biomedcentral.com/content/pdf/14 71-2458-9-190.pdf

22. Oblitas FYM, Loncharich N, Salazar ME, David HML, Silva I, Velásques D. Nursing's role in tuberculosis control: a discussion from the perspective of equity. Rev Lat-am Enfermagem. 2010; 18(1):130-8. 